\title{
TEORIA DA CONFIABILIDADE APLICADA NA AVALIAÇÃO DA VIDA EM FADIGA-DE-CONTATO
}

\section{THEORY OF RELIABILITY APPLIED IN THE EVALUATION OF THE LIFE IN CONTACT FATIGUE}

\author{
Nelson Vanegas M. ${ }^{1} \quad$ John Álvarez R. ${ }^{2} \quad$ Gilberto F. M. de Souza ${ }^{3}$ \\ Recibido 16 de junio de 2008, aceptado 9 de octubre de 2009 \\ Received: June 16, 2008 Accepted: October 9, 2009
}

\begin{abstract}
RESUMO
A confiabilidade é uma ferramenta fundamental no desenvolvimento de projetos cuja aplicação visa a melhoria do desempenho através da redução da probabilidade de falha dos mesmos. Neste estudo empregou-se a teoria de confiabilidade nos critérios de projeto e seleção de ferros fundidos com matrizes de elevada dureza utilizados em diversas aplicações. A partir destes conceitos e das informações geradas em laboratório, em equipamento para ensaios de fadiga de contato de rolamento, avaliou-se a confiabilidade empregando-se os métodos não paramétrico e o paramétrico (com o emprego da distribuição de Weibull), a fim de caracterizar o comportamento da confiabilidade, da probabilidade de falha e da taxa de falha dos dois materiais estudados (ferro fundido cinzento e ferro fundido nodular). Os métodos foram comparados obtendo-se resultados semelhantes, mas foi o método paramétrico (distribuição de Weibull) que melhor modelou o fenômeno em estudo.
\end{abstract}

Palavras-Chave: Confiabilidade, fadiga-de-contato, testes de confiabilidade.

\begin{abstract}
Reliability is a fundamental tool in the development of projects since it allows the improvement of performance through the reduction of the failure probability of the products. In this study the reliability theory was applied as the criteria for design and selection of cast irons with high hardness matrix used in different applications. Based on these concepts and from information generated in laboratory tests, in a rolling contact fatigue testing machine, reliability analysis using non parametric approaches and the Weibull's distribution is developed, in order to characterize reliability, probability of failure, and the rate of failure of both materials (gray cast iron and ductile cast iron). When comparing both methods, similar results were obtained, but the parametric approach (Weibull distribution) has better represented the phenomenon under analysis.
\end{abstract}

Keywords: Reliability, contact fatigue, reliability tests.

\section{INTRODUÇÃO}

A fadiga-de-contato não está unicamente restrita ao estudo das falhas dos rolamentos. Este é um fenômeno que preocupa o setor ferroviário, aos fabricantes de cilindros de laminação, de cames, de engrenagens e também a indústria da fundição, entre outros [1]. Nesta última verifica-se uma crescente tendência à substituição do aço pelo ferro fundido na fabricação de alguns componentes. O ferro fundido é uma material que une excelentes propriedades mecânicas à facilidade de fabricação e, ainda, à elevada resistência ao desgaste [2].

Nesse contexto, insere-se este trabalho, o qual tem por objetivo aplicar a teoria de confiabilidade nos critérios de projeto e seleção de materiais empregados na fabricação de cilindros para a laminação de aços; a fim de quantificar a probabilidade de falha associada à estas ligas, no que se refere ao fenômeno de fadiga-de- contato de rolamento. Com a aplicação destes conceitos busca-se, a partir das

1 Escuela de Ingeniería Eléctrica y Mecánica. Universidad Nacional de Colombia. Carrera 64 \# 63120 Núcleo del Río Bloque 04. Medellín, Colombia. E-mail: nvanegas@unal.edu.co

2 Escola Politécnica. Departamento de Engenharia Mecânica. Universidade de São Paulo. Av. Prof. Mello Moraes, 2231. Cidade Universitária. CEP: 05508-900. São Paulo, Brasil. E-mail: john.rosario@poli.usp.br

3 Escola Politécnica. Departamento de Engenharia Mecatrônica e de Sistemas Mecânicos. Universidade de São Paulo. Av. Prof. Mello Moraes, 2231. Cidade Universitária. CEP: 05508-900. São Paulo, Brasil. E-mail: gfmsouza@ usp.br 
informações geradas em laboratório num equipamento para ensaios de fadiga-de- contato de rolamento, obter informações em termos de probabilidade de falha que permitam ter um critério de vida do componente, como ferramenta para a seleção dos materiais a serem empregados em uma aplicação específica.

Neste trabalho apresentam-se os fundamentos do fenômeno de fadiga-de-contato de rolamento e os fatores que afetam a vida em fadiga-de-contato. São apresentados as características dos ensaios onde os dados do fenômeno em estudo são obtidos e com os quais se realizam os diferentes estudos de confiabilidade que permitem modelar o fenômeno avaliado.

\section{Fenômeno de fadiga-de-contato}

A Figura 1 apresenta-se um conjunto bastante amplo de fatores que interferem no fenômeno de fadiga-de- contato de rolamento.

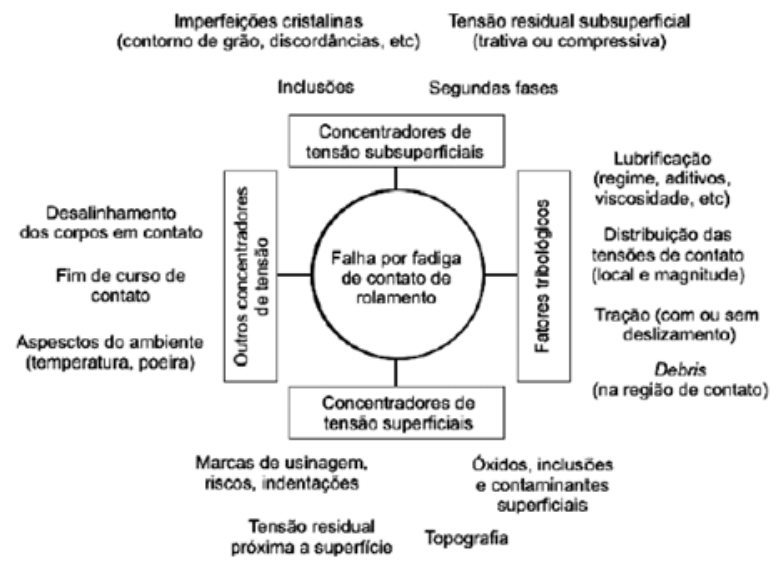

Figura 1. Fatores que afetam a vida em fadiga-de-contato [3].

Nos fenômenos de desgaste por fadiga a primeira distinção a ser feita é entre a fadiga dita convencional, ou seja, à fadiga estrutural relacionada a tensões distribuídas em grandes volumes e à fadiga-de-contato. Essa última está relacionada a esforços localizados, ou seja, distribuídos em áreas que são muito pequenas comparadas às dimensões dos corpos envolvidos. Embora a expressão "fadiga-decontato" seja consensualmente utilizada para referir-se à fadiga-de- contato de rolamento [4], também existem fenômenos de fadiga envolvendo altas tensões de contato que podem ocorrer em situações quase estáticas e de deslizamento.

A fadiga-de-contato decorre do carregamento cíclico sobre contatos não-conformes. Esse carregamento resulta no acúmulo de deformações plásticas que, após um número finito de ciclos, pode conduzir a nucleação de uma trinca [4]. Estão sujeitos a esse fenômeno, entre outros, componentes tais como esferas, rolos e pistas de rolamentos, cames, dentes de engrenagens, cilindros de laminação, trilhos e rodas ferroviárias.

Diferentemente da fadiga convencional, na fadiga-decontato de rolamento não é esperado que exista um limite de carregamento nominal abaixo do qual o componente jamais apresentará falha por fadiga, mesmo para aços [5].

No movimento de rolamento puro de um cilindro sobre uma superfície plana, a máxima tensão de cisalhamento está posicionada abaixo da superfície de contato. No movimento de deslizamento, no entanto, a máxima tensão cisalhante ocorre na superfície. Em regimes mistos de rolamento/deslizamento a posição da máxima tensão cisalhante dependerá da magnitude do atrito de deslizamento. Na Figura 2 estão ilustradas essas três condições, sendo que na combinação de rolamento/ deslizamento, a curva pontilhada mostra que o atrito entre os corpos é suficiente para aproximar a máxima tensão cisalhante da superfície.

As tensões desenvolvidas abaixo da superfície podem então interagir, por exemplo, com alguma inclusão posicionada em seu campo de ação e este ser o ponto inicial de deformação localizada a partir do qual uma trinca será nucleada e se propagará pelo material até o desprendimento de uma lasca da superfície $[6,7,8]$. Se, após a propagação da trinca, for gerada fratura no material, decorrendo no desprendimento de pequenas partículas de material da superfície, o fenômeno é conhecido como "pitting". Geralmente, o pitting é seguido da perda de grandes quantidades de material na superfície, o qual é conhecido como "spalling".

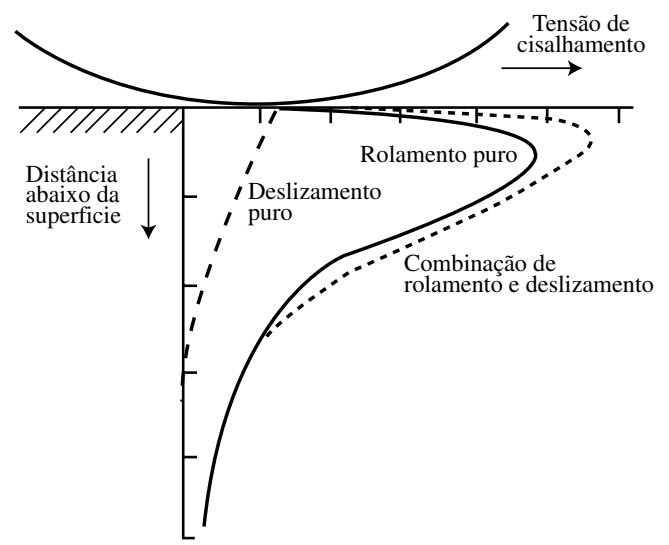

Figura 2. Representação esquemática da distribuição de tensões de cisalhamento abaixo do contato nas situações de rolamento puro [3]. 


\section{MATERIAIS E MÉTODOS}

Nesse estudo foram utilizados dois tipos de ferros fundidos, cinzento e nodular, os quais foram submetidos a tratamentos térmicos de têmpera e revenido com o objetivo de elevar a dureza global dos materiais. Foi empregado um equipamento desenvolvido por Neves [1], para ensaios de fadiga-de- contato, utilizado para a caracterização de ferros fundidos com matrizes de elevada dureza, com o qual se coletaram os dados experimentais, empregando cinco amostras para cada um dos materiais em questão.

\section{Caracterização dos materiais ensaiados}

Os dois tipos de ferros fundidos utilizados nesse trabalho foram fornecidos na forma de barras redondas e lingotadas continuamente. Estes materiais sofreram tratamento térmico de têmpera e revenido. Após o tratamento térmico as duas ligas apresentaram matrizes martensíticas com dureza superior aos $780 \mathrm{HV}$ [1]. As composições das duas ligas são apresentadas na Tabela 1.

Tabela 1. Composições dos ferros fundidos utilizados nos ensaios de fadiga-de-contato [1].

\begin{tabular}{|c|l|c|c|}
\hline \multicolumn{2}{|c|}{ Material } & $\begin{array}{c}\text { Ferro } \\
\text { fundido } \\
\text { cinzento }\end{array}$ & $\begin{array}{c}\text { Ferro } \\
\text { fundido } \\
\text { nodular }\end{array}$ \\
\hline \multirow{4}{*}{$\begin{array}{c}\text { Composiçãa } \\
\text { Quimica [\%] }\end{array}$} & $\mathrm{C}$ & 3,41 & 3,75 \\
\cline { 2 - 4 } & $\mathrm{Si}$ & 2,58 & 2,54 \\
\cline { 2 - 4 } & $\mathrm{Mn}$ & 0,30 & 0,16 \\
\cline { 2 - 4 } & $\mathrm{P}$ & 0,068 & 0,068 \\
\cline { 2 - 4 } & $\mathrm{S}$ & 0,086 & 0,009 \\
\cline { 2 - 4 } & $\mathrm{Mg}$ & 0,15 & 0,60 \\
\cline { 2 - 4 } & $\mathrm{Cr}$ & 0,001 & 0,037 \\
\cline { 2 - 4 } & $\mathrm{Ni}$ & 0,075 & 0,058 \\
\cline { 2 - 4 } & $\mathrm{Ti}$ & 0,012 & 0,020 \\
\cline { 2 - 4 } & $\mathrm{V}$ & 0,007 & 0,011 \\
\cline { 2 - 4 } & $\mathrm{Nb}$ & 0,008 & 0,008 \\
\cline { 2 - 4 } & $\mathrm{CE}$ & 4,06 & 4,005 \\
\hline
\end{tabular}

\section{Condições gerais de ensaio}

Os experimentos foram realizados por Neves [1], sendo conduzidos com lubrificação e sob mesma de tensão nominal de contato entre os dois materiais, ferro fundido cinzento e ferro fundido nodular. Como lubrificante foi utilizado um óleo comercial para caixa de engrenagens com designação SAE 90 API-GL4. A tensão de contato máxima 3,6GPa, foi obtida mediante o carregamento do braço de carga da máquina com pesos mortos de $245 \mathrm{~N}$. Esses carregamentos resultaram em cargas totais de ensaio de $1146 \mathrm{~N}$.
O parâmetro de término de cada ensaio foi determinado pela ocorrência do primeiro lascamento da trilha de rolamento, o que foi detectado pelo aumento abrupto do ruído emitido pelo equipamento de ensaio [1]. As condições de ensaio são sumarizadas na Tabela 2.

Tabela 2. Condições de ensaio [1].

\begin{tabular}{|l|l|}
\hline Lubrificante & $\begin{array}{l}\text { SAE 90 } \\
\text { API-GL4 }\end{array}$ \\
\hline Temperatura do lubrificante & $50 \pm 2{ }^{\circ} \mathrm{C}$ \\
\hline Carga total & $1146 \mathrm{~N}$ \\
\hline Número de esferas & 3 \\
\hline Carga por esfera & $382 \mathrm{~N}$ \\
\hline Diâmetro da esfera & $7,398 \mathrm{~mm}$ \\
\hline Módulo combinado & $101,2 \mathrm{GPa}$ \\
\hline Raio do contato & $0,224 \mathrm{~mm}$ \\
\hline Área de contato & $0,158 \mathrm{~mm}^{2}$ \\
\hline Pressão média de contato $(\mathrm{pm})$ & $2,4 \mathrm{GPa}$ \\
\hline Máxima pressão de contato $(\mathrm{po})$ & $3,6 \mathrm{GPa}$ \\
\hline Frequiência de carregamento & $46 \mathrm{~Hz}$ \\
\hline
\end{tabular}

\section{Ensaios de fadiga-de-contato de rolamento}

Neste grupo de resultados encontram-se os valores obtidos para todos os corpos-de-prova, ou seja, os resultados dos ensaios com os dois materiais testados. Na Tabela 3 são apresentadas as vidas em fadiga obtidas para cada corpo-de-prova com os dois materiais sob as condições gerais de ensaio.

Tabela 3. Vidas, em ciclos de carregamento, dos corpos-deprova nos ensaios de fadiga-de-contato [1].

\begin{tabular}{|c|c|c|}
\hline $\begin{array}{c}\text { No de } \\
\text { ordem (i) }\end{array}$ & $\begin{array}{c}\text { Ferro fundido } \\
\text { cinzento } \\
\text { [ciclos] }\end{array}$ & $\begin{array}{c}\text { Ferro fundido } \\
\text { nodular } \\
\text { [ciclos] }\end{array}$ \\
\hline 1 & 107.000 & 609.000 \\
\hline 2 & 88.000 & 1.296 .000 \\
\hline 3 & 43.000 & 1.602 .000 \\
\hline 4 & 90.000 & 1.385 .000 \\
\hline 5 & 76.000 & 1.584 .000 \\
\hline
\end{tabular}

\section{RESULTADOS}

Neste trabalho foram empregadas duas formas de análise de confiabilidade que possibilitaram, através de estimativa, caracterizar os comportamentos da confiabilidade, da probabilidade da falha e da taxa de falha dos dois materiais expressas em função do número de ciclos de carregamento. Os dois testes de confiabilidade foram:

- Teste não-paramétrico.

- Teste paramétrico. 


\section{Testes não-paramétrico para o ferro fundido cinzento}

É o primeiro passo para caracterizar um experimento, sob o ponto de vista da abordagem em confiabilidade, fornecendo uma estimativa relativamente rápida e de baixo custo para as funções de interesse. Com os dados não-agrupados dos ciclos até a falha, apresentados na Tabela 3, estimou-se a confiabilidade, a função densidade de probabilidade dos ciclos até a falha e a função taxa de falha, traçando posteriormente os gráficos respectivos para cada uma delas. Calculou-se também o mean time to failure (MTTF) e a variância.

Foram empregadas as seguintes equações:

- Para determinar as estimativas de confiabilidade:

$$
\hat{R}\left(x_{i}\right)=(N+1-i) /(N+1) \operatorname{com} N=5
$$

- Para determinar as estimativas da função densidade de probabilidade:

$$
\hat{f}(x)=1 /\left[\left(x_{i+1}-x_{i}\right)(N+1)\right]
$$

- E para determinar as estimativas da taxa de falha:

$$
\hat{\lambda}(x)=\hat{f}(x) / \hat{R}(x)
$$

Os resultados das estimativas da confiabilidade, da função densidade de probabilidade e da taxa de falha são apresentados, de forma gráfica, nas Figuras 3, 4 e 5 respectivamente.

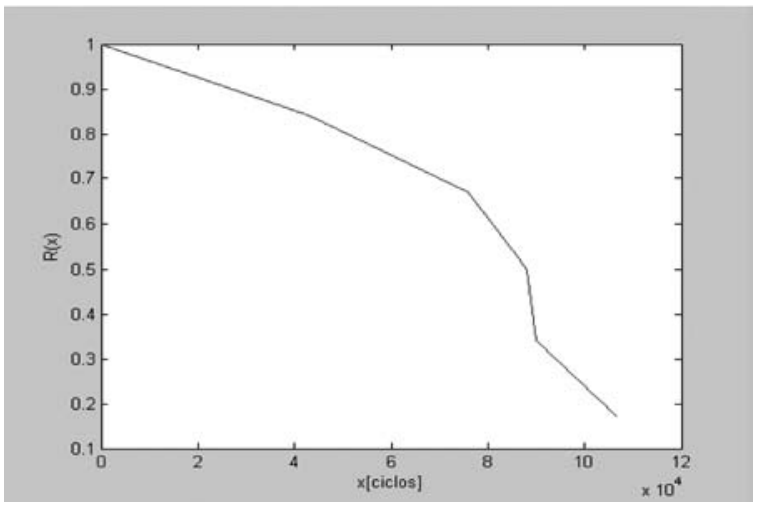

Figura 3. Estimativa da confiabilidade, teste nãoparamétrico para o ferro fundido cinzento.

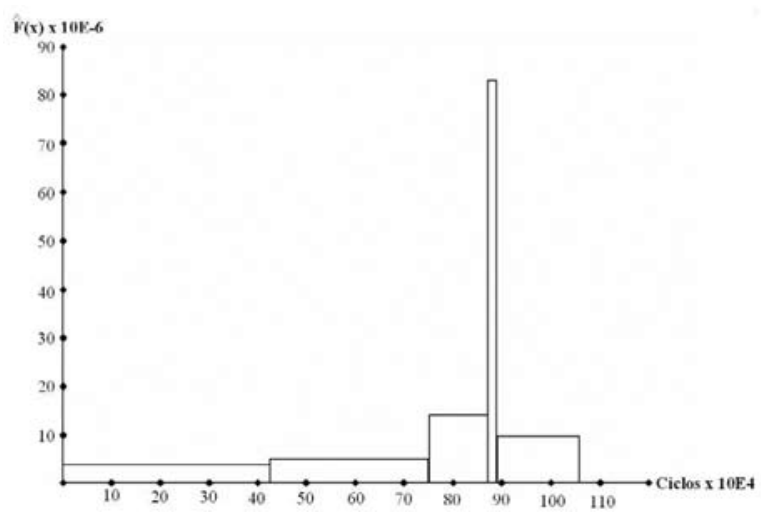

Figura 4. Estimativa da função densidade de probabilidade, teste não-paramétrico para o ferro fundido cinzento.

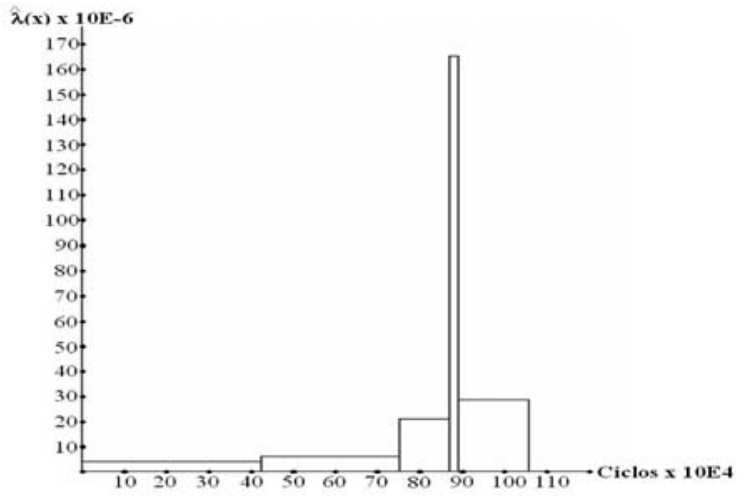

Figura 5. Estimativa da taxa de falha, teste não-paramétrico para o ferro fundido cinzento.

A partir da observação destes dados pode-se afirmar que a função densidade de probabilidade do processo em estudo apresenta um pico bem definido, pode ser considerada assimétrica, assemelhando-se a uma distribuição de Weibull.

A curva da taxa de falha indica que esta é crescente ao longo do tempo, indicando que o mecanismo de falha é cumulativo ao longo do tempo de operação, embora se verifique um comportamento decrescente ao final da curva. Talvez este fenômeno seja devido a dois dados do experimento que estão muito próximos (88.000 e 90.000 ciclos).

Calculou-se o ciclo médio até a falha com o emprego da eq. (4):

$$
\hat{m}_{x}=(1 / N)\left(\sum_{i=1}^{N} x_{i}\right), \text { com } N=5
$$

$\hat{m}_{x}=(1 / 5)(404.000) \Rightarrow \hat{m}_{x}=80.800$ ciclos 
Ou seja, a ciclagem média até a falha é 80.800 ciclos.

A variância da ciclagem até a falha é estimada pela eq. (5):

$$
\begin{aligned}
\hat{\sigma}_{x}^{2} & =[1 /(N-1)]\left[\sum_{i=1}^{N}\left(x_{i}-\hat{m}_{x}\right)\right], \text { com } N=5 \\
\Rightarrow & \hat{\sigma}_{x}^{2}=5.687 x 10^{5} \Rightarrow \hat{\sigma}_{x}=23.847,43 \text { ciclos }
\end{aligned}
$$

Ou seja, a variância do processo é de $5.687 \times 10^{5}$ ciclos, e o desvio padrão é de 23.847 ciclos.

\section{Teste paramétrico para o ferro fundido cinzento}

A partir dos dados coletados experimentalmente, foram estimados os parâmetros da distribuição de Weibull que supõe-se caracterizar o comportamento do processo em estudo, se determinando a melhor representação estatística da função de confiabilidade e desta forma modelar o fenômeno em estudo.

Para a determinação da média e do desvio padrão da distribuição de vida dos componentes ensaiados é necessária a estimação dos parâmetros da distribuição de Weibull que rege o mecanismo de falha em estudo.

Os dados de ciclos até a falha são ordenados em ordem crescente, executando-se a estimativa da função distribuição acumulada para cada tempo de falha, utilizando a eq. (6), a qual é apresentada na Tabela 4.

$$
\hat{F}\left(x_{i}\right)=i /(N+1)
$$

Tabela 4. Estimativa da função distribuição acumulada para o ferro fundido cinzento.

\begin{tabular}{|c|c|c|}
\hline$i$ & $x_{i}$ & $\hat{F}\left(x_{i}\right)$ \\
\hline 1 & 43.000 & 0,17 \\
\hline 2 & 76.000 & 0,33 \\
\hline 3 & 88.000 & 0,50 \\
\hline 4 & 90.000 & 0,67 \\
\hline 5 & 107.000 & 0,83 \\
\hline
\end{tabular}

Posteriormente, cada um dos pares ordenados $\left(x_{i}, \hat{F}\left(x_{i}\right)\right)$ é marcado no papel de probabilidade da distribuição de Weibull [9], apresentado na Figura 6.

Verifica-se que os pontos em questão tendem a se distribuir conforme uma linha reta, ou seja, o fenômeno pode ser representado mediante uma distribuição de Weibull.

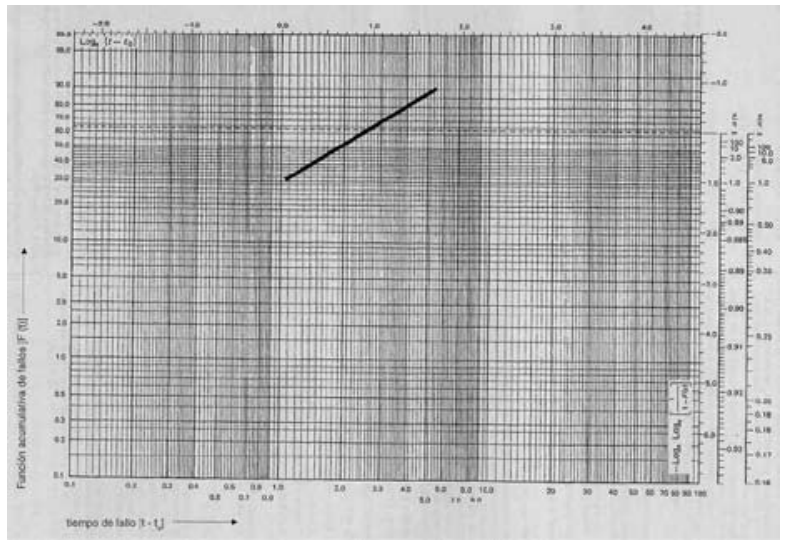

Figura 6. Distribuição de Weibull para o ferro fundido cinzento.

Os parâmetros que regem a distribuição de Weibull são definidos pelas relações:

- Parâmetro $\eta: \eta=27.000$ ciclos

- Parâmetro $\beta$ : $\beta=1,2$

- $\operatorname{Média}\left(\hat{m}_{x}\right)$

$$
\left(\hat{m}_{x}-x_{0}\right) / \eta=0,968 \Rightarrow \hat{m}_{x}=90.836 \text { ciclos }
$$

- Desvio padrão $\left(\hat{\sigma}_{x}\right)$

$$
\hat{\sigma}_{x} / \eta=0,78 \Rightarrow \hat{\sigma}_{x}=21.060 \text { ciclos }
$$

Deve-se ressaltar que os valores obtidos para os parâmetros da distribuição são também estimativos, uma vez que são determinados com base na análise de dados geraos a partir das amostras ensaiadas.

Com esta análise finaliza-se o processo de definição de parâmetros, e pode-se afirmar que a confiabilidade do material ferro fundido cinzento é regida pela seguinte distribuição:

$$
R(x)=e^{\left\{-((x-64.700) / 27.000)^{1,2}\right\}}
$$

sendo que a vida média do material é 90.836 ciclos e o desvio padrão é 21.060 ciclos. O material apresenta uma vida mínima de 64.700 ciclos.

O expoente da distribuição é de 1,2. Nesta situação a distribuição de Weibull esta indicando um processo que tem taxa de falha crescente no tempo, ou seja, o mecanismo de falha dominante é do tipo cumulativo [10].

Na Tabela 5, apresenta-se uma comparação dos resultados obtidos empregando os dois testes de confiabilidade no estudo do fenômeno de fadiga de contato do ferro fundido cinzento. 
Tabela 5. Comparação dos resultados do teste nãoparamétrico e do teste paramétricos para caracterizar o ferro fundido cinzento.

\begin{tabular}{|l|c|c|}
\hline \multicolumn{1}{|c|}{ Parâmetro } & $\begin{array}{c}\text { Teste } \\
\text { não-paramétrico }\end{array}$ & $\begin{array}{c}\text { Teste } \\
\text { paramétrico }\end{array}$ \\
\hline $\begin{array}{l}\text { Média }\left(\hat{m}_{x}\right) \\
\text { [ciclos] }\end{array}$ & 80.800 & 90.836 \\
\hline $\begin{array}{l}\text { Desvio padrão } \\
\left(\hat{\sigma}_{x}\right) \text { [ciclos] }\end{array}$ & 23.847 & 21.060 \\
\hline$\eta$ [ciclos] & - & 27.000 \\
\hline$\beta$ & - & 1,2 \\
\hline $\begin{array}{l}\text { Vida mínima } \\
\text { [ciclos] }\end{array}$ & - & 64.700 \\
\hline
\end{tabular}

\section{Testes não-paramétrico para o ferro fundido nodular}

De forma semelhante ao executado com o primeiro material, no ferro fundido nodular foram realizados dois testes de confiabilidade.

Conforme a Tabela 3, estimou-se a confiabilidade, a função densidade de probabilidade dos ciclos até a falha e a função taxa de falha, traçando posteriormente os gráficos respectivos para cada uma delas. Calculou-se também o MTTF e a variância.

Foram empregadas as equações: eq. (1), eq. (2) e eq. (3) para determinar as estimativas de confiabilidade, da função densidade de probabilidade e da taxa de falha respectivamente.

Os resultados das estimativas da confiabilidade, a função densidade de probabilidade e taxa de falha são apresentados, de forma gráfica, nas Figuras 7, 8 e 9 respectivamente.

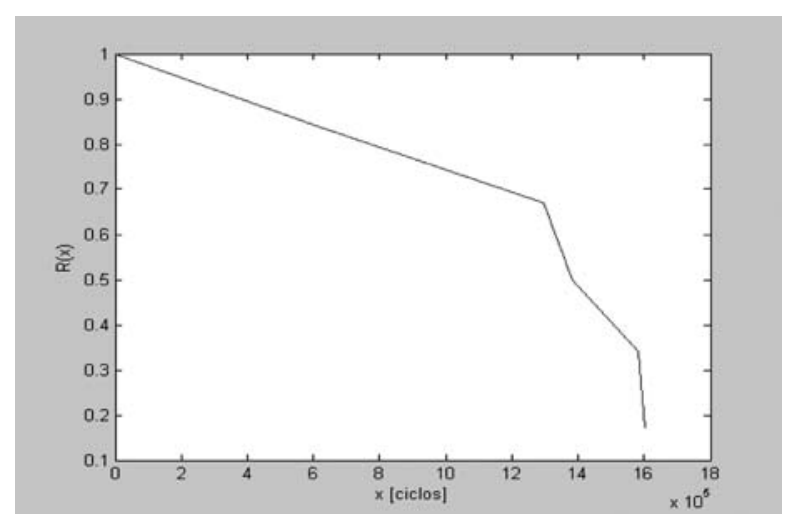

Figura 7. Estimativa da confiabilidade, teste nãoparamétrico para o ferro fundido nodular.

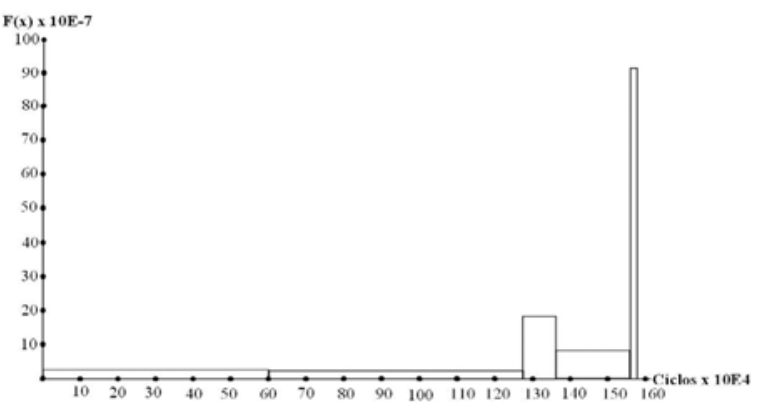

Figura 8. Estimativa da função densidade de probabilidade, teste não-paramétrico para o ferro fundido nodular.

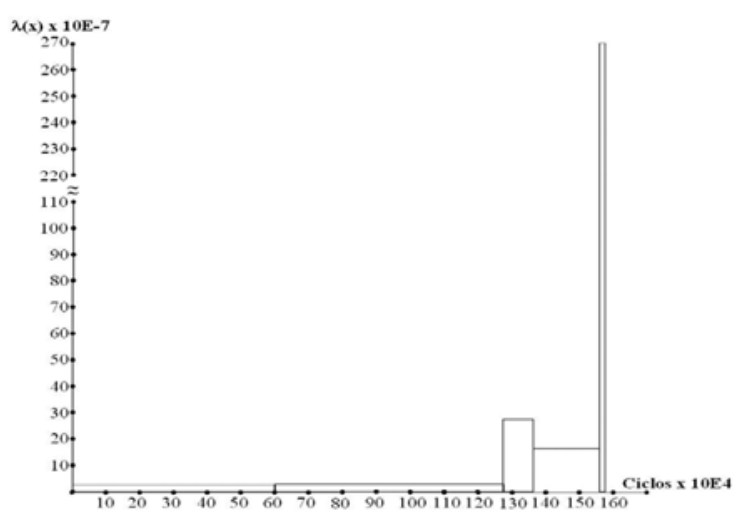

Figura 9. Estimativa da taxa de falha, teste não-paramétrico para o ferro fundido nodular.

Observando os dados pode-se afirmar que a função densidade de probabilidade do processo em estudo apresenta um pico bem definido, e pode-se dizer que tende a uma distribuição de Weibull.

A curva da taxa de falha indica que esta é crescente ao longo do tempo, indicando que o mecanismo de falha é cumulativo ao longo do tempo de operação. Verifica-se um pico muito alto no final da curva da taxa de falha, sendo este fenômeno asociado ao fato dos dois últimos dados do experimento estarem muito próximos (1.584.000 e 1.602 .000 ciclos).

Calculou-se o ciclo médio até a falha com o emprego da eq. (4):

$\hat{m}_{x}=(1 / 5)(6.476 .000) \Rightarrow \hat{m}_{x}=1.295 .200$ ciclos

Ou seja, a ciclagem média até a falha é 1.295.200 ciclos.

A variância da ciclagem até a falha é estimada pela eq. (5):

$\hat{\sigma}_{x}^{2}=1,64 \times 10^{11} \Rightarrow \hat{\sigma}_{x}=404.660,35$ ciclos 
Ou seja, a variância do processo é de $1,64 \times 10^{11}$ ciclos, e o desvio padrão é de 404.660 ciclos.

\section{Teste paramétrico para o ferro fundido nodular}

Para a determinação da média e do desvio padrão da distribuição da vida dos componentes ensaiados foi necessária a estimação dos parâmetros da distribuição de Weibull que rege o mecanismo de falha em estudo.

Os dados de ciclos até a falha são ordenados em ordem crescente, executa-se a estimativa da função distribuição acumulada para cada tempo de falha, utilizando a eq. (6), e obtendo-se a Tabela 6.

Tabela 6. Estimativa da função distribuição acumulada para o ferro fundido nodular.

\begin{tabular}{|c|c|c|}
\hline$i$ & $x_{i}$ & $\hat{F}\left(x_{i}\right)$ \\
\hline 1 & 609.000 & 0,17 \\
\hline 2 & 1.296 .000 & 0,33 \\
\hline 3 & 1.385 .000 & 0,50 \\
\hline 4 & 1.584 .000 & 0,67 \\
\hline 5 & 1.602 .000 & 0,83 \\
\hline
\end{tabular}

Posteriormente cada um dos pares ordenados $\left(x_{i}, \hat{F}\left(x_{i}\right)\right)$ é marcado no papel de probabilidade da distribuição de Weibull [9], apresentado na Figura 10.

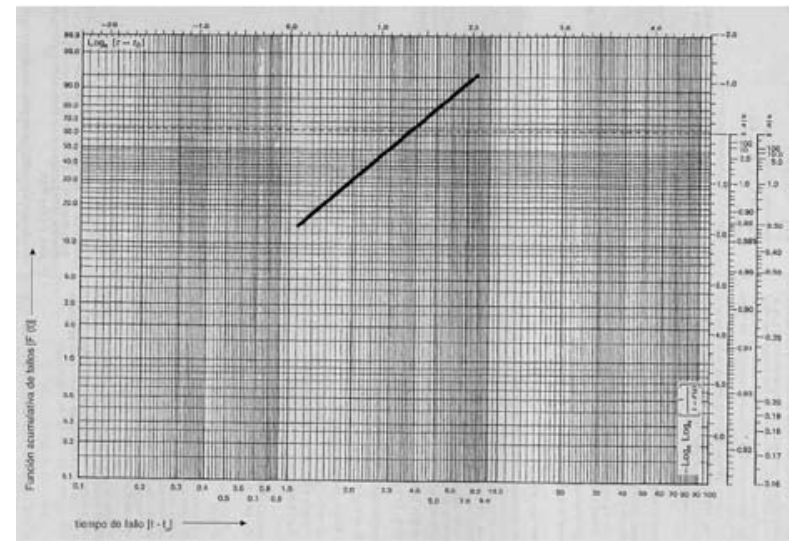

Figura 10. Distribuição de Weibull para o ferro fundido nodular.

Verifica-se que estes tendem a se distribuir conforme uma linha reta, ou seja, o fenômeno pode ser representado por uma distribuição de Weibull.

Os parâmetros que regem a distribuição de Weibull são definidos pelas relações:
- Parâmetro $\eta: \eta=410.000$ ciclos

- Parâmetro $\beta: \beta=1,41$

- Média $\left(\hat{m}_{x}\right)$ $\left(\hat{m}_{x}-x_{0}\right) / \eta=0,92 \Rightarrow \hat{m}_{x}=1.455 .200$ ciclos

- Desvio padrão $\left(\hat{\sigma}_{x}\right)$ $\hat{\sigma}_{x} / \eta=0,63 \Rightarrow \hat{\sigma}_{x}=258.300$ ciclos

Como já apresentado no caso da análise do teste paramétrico para o ferro fundido cinzento, deve-se ressaltar que os valores obtidos para os parâmetros da distribuição são também estimativos.

Com esta análise finaliza-se o processo de definição de parâmetros, e pode-se afirmar que a confiabilidade do material ferro fundido nodular é regida pela seguinte distribuição:

$$
R(x)=e^{\left\{-((x-1.078 .000) / 410.000)^{1,41}\right\}}
$$

sendo que a vida média do material é 1.455 .200 ciclos e do desvio padrão é 258.300 ciclos. O material apresenta uma vida mínima de 1.078 .000 ciclos.

O expoente da distribuição é de 1,41. Nesta situação a distribuição de Weibull esta indicando um processo que tem taxa de falha crescente no tempo [10], ou seja, o mecanismo de falha dominante é do tipo cumulativo.

Na Tabela 7, apresenta-se uma comparação dos resultados obtidos empregando os dois testes de confiabilidade para o estudo do fenômeno de fadiga-de- contato do ferro fundido nodular.

Tabela 7. Comparação dos resultados do teste nãoparamétrico e do teste paramétrico para caracterizar o ferro fundido nodular.

\begin{tabular}{|l|c|c|}
\hline \multicolumn{1}{|c|}{ Parâmetro } & $\begin{array}{c}\text { Teste } \\
\text { não-paramétrico }\end{array}$ & $\begin{array}{c}\text { Teste } \\
\text { paramétrico }\end{array}$ \\
\hline $\begin{array}{l}\text { Média }\left(\hat{m}_{x}\right) \\
\text { [ciclos] }\end{array}$ & 1.295 .200 & 1.455 .200 \\
\hline $\begin{array}{l}\text { Desvio padrão } \\
\left(\hat{\sigma}_{x}\right) \text { [ciclos] }\end{array}$ & 404.660 & 258.300 \\
\hline$\eta$ [ciclos] & - & 410.000 \\
\hline$\beta$ & - & 1,41 \\
\hline $\begin{array}{l}\text { Vida mínima } \\
\text { [ciclos] }\end{array}$ & - & 1.078 .000 \\
\hline
\end{tabular}

As estimativas obtidas pelos dois métodos apresentam valores muito próximos sendo que o teste não-paramétrico caracteriza o experimento gerando uma estimativa muito 
rápida, já o teste paramétrico envolvendo a estimativa dos parâmetros da distribuição de Weibull empregando o método gráfico foi mais dispendioso, mas gerou parâmetros mais claros que permitem estimar o comportamento da confiabilidade, da probabilidade de falha e da taxa de falha.

\section{CONCLUSÕES}

Baseado na observação dos dados, para os materiais estudados, ferro fundido cinzento e ferro fundido nodular, pode-se afirmar que a função densidade de probabilidade dos processos em estudo apresenta um pico bem definido, e foram melhor modelados com uma distribuição de Weibull. A taxa de falha é crescente com o aumento da magnitude do número de ciclos de operação, representando a fase III da curva da banheira, confirmando a seleção da distribuição de Weibull em função do comportamento da taxa da falha. Este fato é confirmado à obter o parâmetro $\beta$ com valor maior que um $(\beta>1)$. Este resultado era esperado pois o fenômeno de fadiga é um processo cumulativo tendo em vista a natureza do mecanismo de nucleação e propagação da trinca.

Os testes não-paramétricos em ambos os materiais apresentam valores de média e desvio padrão muito similares aos obtidos através da análise da distribuição de Weibull.

A estimativa dos parâmetros pela distribuição de Weibull, permite caracterizar o problema em estudo para ambos os materiais. $\mathrm{O}$ parâmetro $\beta$ foi maior para o ferro fundido nodular $(1,41)$ quando comparado com o ferro fundido cinzento $(1,2)$, ou seja, este último apresenta uma maior vida em fadiga.

Conforme apresentado nas Tabelas 5 e 7, os dois testes de confiabilidade empregados permitiram obter estimativas dos parâmetros que caracterizam o fenômeno de fadigade-contato com valores muito próximos.

Comparativamente o ferro fundido nodular apresentou valores de taxa de falha menores que o ferro fundido cinzento. Igualmente, pode-se afirmar ao comparar as figuras da função acumulada da distribuição de Weibull de ambos os materiais, que o ferro fundido nodular apresentou um melhor desempenho do material, o qual está relacionado com às características microestruturais da liga. Desta forma, empregou-se a teoria de confiabilidade como critério de projeto e seleção de ligas ao se comparar ambos os ferros fundidos, e conclui-se que o ferro fundido nodular apresenta um desempenho satisfatório na vida à fadiga-de-contato, e pode ser utilizado para a fabricação de componentes que precisam atender a este tipo de solicitação mecânica.

Os dados levantados por Neves [1] permitiram quantificar o desempenho do ferro fundido nodular e do ferro fundido cinzento quando submetidos a fadiga-de- contato de rolamento. Estas informações depois de serem analisadas mediante a teoria de confiabilidade, permitiram determinar gráfica e analiticamente o comportamento da taxa de falha e da função densidade de probabilidade para os materiais em questão. Visando a importância tecnológica das outras famílias de ferros fundidos existentes, recomenda-se realizar experiências com ferros fundidos brancos e ferros fundidos mesclados, no intuito de aplicar a metodologia como critério para a seleção e comparação da confiabilidade desses materiais quando submetidos à fadiga-de-contato de rolamento.

\section{REFERÊNCIAS}

[1] J.C.K. das Neves. "Desenvolvimento de um Equipamento para Ensaio de Fadiga de Contato Esfera Sobre Plano e sua Aplicação na Caracterização de Ferros Fundidos com Matrizes de Elevada Dureza”. Tese Escola Politécnica. Universidade de São Paulo. São Paulo, Brasil. 2006.

[2] L. Magalhães, J. Seabra and C. Sá. "Experimental observations of contact fatigue crack mechanisms for austempered ductile iron (ADI) discs". J. Wear. Vol. $246 \mathrm{~N}^{\circ}$ 1, pp. 134-148. 2000.

[3] S.R. Lampman. "ASM Handbook. Fatigue and fracture". ASM International. Vol. 19, p. 1057. 1996.

[4] G.W. Stachowiak, A.W. Batchelor and G. Stachowiak. "Engineering Tribology". Butterworth Heinemann. Boston, p. 832. 2005.

[5] E. Rabinowicz. "Friction and Wear of Materials". John Wiley \& Sons. 2 edition, p. 332. New York, Estados Unidos. 1995.

[6] D. Girodin, F. Ville, R. Guers and G. Dudragne. "Rolling contact fatigue test to investigate surface initiated damage and tolerance to surface dents". Bearing Steel Technology. ASTM STP 1419, J.M. Beswick. Ed. American Society for Testing and Materials International. West Conshohocken, P.A, pp. 263-281. 2002. 
[7] R.F. Johnston and J.F. Sewell. "The bearing properties of $1 \% \mathrm{C}$-Cr steel as influenced by steelmaking practice". Journal of Iron and Steel Institute. Vol. 196 part 4, pp. 414-444. December 1960.

[8] T.E. Tallian and J.I. McCool. "An engineering model of spalling fatigue failure in rolling contact, II". The Surface Model, Wear. Vol.17, pp. 447-461. 1971.

[9] E.E. Lewis. "Introduction to Reliability Engineering". Jhon Wiley \& Sons. Department of Mechanical and Nuclear Engineering Northwestern University. Second edition, p. 464. New York, Estados Unidos. 1996.

[10] G.F.M. de Souza. "Análise de Confiabilidade Aplicada ao Projeto de Sistemas Mecânicos". Escola Politécnica da Universidade de São Paulo. Departamento de Engenharia Mecatrônica e Sistemas Mecânicos, p. 210. São Paulo, Brasil. 2008. 